PAPER

\section{Pressure sensitivity of dislocation density in copper single crystals at submicron scale}

To cite this article: Jianqiao Hu et al 2018 Mater. Res. Express 5016504

View the article online for updates and enhancements.
Related content

Avalanches and plastic flow in crystal plasticity: An overview

Stefanos Papanikolaou, Yinan Cui and

Nasr Ghoniem

Primary combination of phase-field and discrete dislocation dynamics methods for investigating athermal plastic deformation in various realistic Ni-base single crystal superalloy microstructures Siwen Gao, Mohan Kumar Rajendran, Marc Fivel et al.

A 3D dislocation dynamics analysis of the size effect on the strength of [ $\left[\begin{array}{lll}1 & 1 & 1\end{array}\right] \mathrm{LiF}$ micropillars at $300 \mathrm{~K}$ and $600 \mathrm{~K}$ Hyung-Jun Chang, Javier Segurado, Jon M Molina-Aldareguía et al. 


\section{Materials Research Express}

\section{PAPER}

CrossMark

\section{Pressure sensitivity of dislocation density in copper single crystals at}

RECEIVED

2 November 2017

REVISED

7 December 2017

ACCEPTED FOR PUBLICATION

11 December 2017

PUBLISHED

4 January 2018

\section{submicron scale}

\author{
Jianqiao $\mathrm{Hu}^{1}\left({ }^{1}\right.$, Zhen Chen ${ }^{2,3,5}$, Zhanli Liu ${ }^{4}$ and Zhuo Zhuang ${ }^{4,5}$ \\ 1 State Key Laboratory of Nonlinear Mechanics, Institute of Mechanics, Chinese Academy of Sciences, Beijing 100190, People's Republic of \\ China \\ 2 International Research Center for Computational Mechanics, State Key Laboratory of Structural Analysis for Industrial Equipment, \\ Department of Engineering Mechanics, Dalian University of Technology, Dalian 116023, People's Republic of China \\ 3 Department of Civil and Environmental Engineering, University of Missouri, Columbia, MO 65211, United States of America \\ 4 Applied Mechanics Lab., School of Aerospace Engineering, Tsinghua University, Beijing 100084, People's Republic of China \\ Authors to whom any correspondence should be addressed. \\ E-mail: chenzh@missouri.edu and zhuangz@tsinghua.edu.cn
}

Keywords: dislocation evolution, hydrostatic pressure, discrete dislocation dynamics

\begin{abstract}
It is known that the mechanical responses of metallic samples are insensitive to confining pressure at macroscale. As a result, von Mises elastoplasticity has been commonly used to model metals in engineering practice. With the use of discrete dislocation dynamics in this study, we explore the dislocation behavior of finite-sized copper single crystals of different sizes under uniaxial compression and hydrostatic pressure, respectively. It is found that the dislocation density approaches a stable value with the increase of hydrostatic pressure while it still keeps increasing under uniaxial compression as the size-dependent yield stress is reached. This difference is also dependent on the loading rate. The yield stress under uniaxial compression exhibits the conventional loading rate effect, while the stable value of dislocation density under hydrostatic compression increases with the increase of loading rate. Moreover, a transition from being pressure-insensitive to pressure-sensitive on the evolution of dislocation density is observed under hydrostatic compression as the sample size becomes small. These findings provide useful insights into the elastoplastic responses of metallic samples at microscale.
\end{abstract}

\section{Introduction}

The mechanical behavior of crystals depends on the dislocation motion in response to the applied loading. Since the mechanical responses at submicron scale differ from those at the macro scale, a fundamental understanding of the size dependency has attracted a lot of attention and investigation [1, 2]. In particular, Cui et al [3] investigated the dislocation evolution in single crystalline copper pillars of different sizes under uniaxial compression, and revealed that it was the single-arm source mechanism controlling the plastic flow behavior for submicron face-centered cubic (fcc) single crystals. Most of the investigations in the open literature have concentrated on the mechanical behavior and corresponding dislocation evolution under uniaxial compression [4-6]. However, the mechanical behaviors along other loading paths might exhibit some different features. For instance, the size effect on the response to hydrostatic pressure, which is rarely studied, might have valuable potential applications under extreme environments such as deep-sea operation and 3D printing of metallic parts. The investigation on the mechanical behavior under hydrostatic pressure is also valuable to better understand the plate impact loading process in which a hydrostatic state of stress becomes dominant [7-9]. In addition, Yuan et al [10] investigated the interplay between pressure and grain size with a series of large-scale molecular dynamics simulations, which showed different dislocation behaviors below and above a critical hydrostatic pressure. The recent work by Chen et al [11] explored the effect of hydrodynamic compression rate on the crystal formation from a noble fluid that contains a $\mathrm{Cu}$ inclusion. Hence, an important issue arises about whether the $\mathrm{Cu}$ inclusion of different sizes would exhibit different mechanical behaviors under hydrostatic 
compression. Since the mechanical responses of metallic samples are insensitive to confining pressure at macroscale, von Mises elastoplasticity has been commonly used for modeling metals in engineering practice. To clarify the applicability of von Mises elastoplasticity at microscale, we use discrete dislocation dynamics (DDD) in this work to explore the mechanical response and corresponding dislocation evolution under hydrostatic pressure. The focus of this work is on the effects of hydrostatic pressure, loading rate and sample size on the evolution of dislocation microstructure.

\section{Simulation details}

The DDD model employed here has been described in our previous studies $[5,12,13]$. The calculation procedure always follows the same general form. The forces acting on all the dislocation segments are evaluated at each time step, dislocation velocities are then calculated by solving the dislocation motion equation, unlike the widely used overdamped linear force-velocity equation for dislocation motion, a fully dynamic equation including inertial effect $[14,15]$ is adopted in the DDD simulation, which has been verified to be more consistent with the experimental results. Finally, the dislocation positions and topology are updated every time step to deal with dislocation surface annihilation and the short range interactions between perfect non-dissociated dislocations. The geometrical boundary change of the computational specimen is not considered here for simplicity.

In this study, a series of DDD simulations of hydrostatic compression test are performed on the finite-sized $\mathrm{Cu}$ single crystalline samples. Each sample is set to a cube with the same edge length ranging from 200 to $1200 \mathrm{~nm}$ in its three-dimensions. These cubes are set with $x$-, $y$-, and $z$-axes along [100], [010], and [001], respectively. As a reference, the uniaxial compression test is also carried out on all the Cu samples to investigate the effect of loading mode. It is worth mentioning that other loading paths such as biaxial compression and triaxial compression (applying hydrostatic compression first, and then keeping compressive loading only along one direction with the loading level in other directions being fixed) are also important. Since this investigation is focused on hydrostatic loading, a detailed study on the other two loading paths is not conducted in this work. All the simulations are performed under stress-controlled compression loading. The material properties are as follows: shear modulus $\mu$ is $48 \mathrm{GPa}$, Poisson's ratio $\nu$ is 0.34 , the mass density $\rho_{c}$ is $8.96 \times 10^{3} \mathrm{~kg} \mathrm{~m}^{-3}$, the magnitude of the Burgers vector $b$ is $0.256 \mathrm{~nm}$, and the viscous drag coefficient $B$ is $2 \times 10^{-5} \mathrm{~Pa}$.

The initial equilibrium dislocation configurations are generated via a relaxation procedure, which approximates a real thermal annealing process $[2,16]$. This process starts with the generation of randomly created straight dislocation lines and internal dislocation loops spreading on all the twelve slip systems of fcc. The ends of straight dislocation lines terminate at the free surface. All the internal dislocations then evolve dynamically without external loading until the structures become stable. More details can be found in [3]. According to the experimental observations $[17,18]$, the mechanical response of micropillars with the same size also varies. This motivates us to use different initial dislocation configurations for each sample size $D$. As a result, the initial dislocation structure and density are different in the $\mathrm{Cu}$ samples from each other. After the initial relaxation procedure, the hydrodynamic compression loading rates ranging from 0.1 to $1.0 \mathrm{MPa} \mathrm{ps}^{-1}$ are applied on all the three-dimensions of cube, i.e. along [100], [010] and [001] crystal orientations. While for the case of uniaxial compression, the same compression loading rates are applied on the top of the cube along [001] crystal orientation, and the lateral surfaces are traction free. The time step used in the simulations is $\Delta t=1 \mathrm{ps}$ which ensures that the numerical results are converged. To compare the responses between hydrostatic compression and uniaxial compression in the simulation results, the stress in hydrostatic compression is defined as $\sigma=-\left(\sigma_{x x}+\sigma_{y y}+\sigma_{z z}\right) / 3$, and the one in the uniaxial loading is $\sigma=-\sigma_{z z}$, for which the corresponding strains are both defined as $\varepsilon=-\left(\varepsilon_{x x}+\varepsilon_{y y}+\varepsilon_{z z}\right)$.

\section{Results and discussion}

\subsection{Effect of loading mode}

In this section, the copper cubes with the edge length $400 \mathrm{~nm}$ are loaded under two different modes, i.e., the uniaxial and hydrostatic compression, respectively. The stress-strain curves and the evolution of dislocation density under the two loading modes are plotted for the $\mathrm{Cu}$ samples with the same size and three different initial configurations, as shown in figure 1. Particularly, different curve types (i.e. solid, dotted and dashed lines) are used to distinguish the different initial dislocation configurations and indicate the correspondence between the evolution of dislocation density and mechanical response. It can be seen from figure 1(a) that the stress-strain curves of $\mathrm{Cu}$ cubes are almost the same under hydrostatic compression when the edge length is $400 \mathrm{~nm}$, for which the stress increases approximately linearly with the strain. Hence, the initial dislocation structures have a limited effect on the mechanical behavior, and the dislocation density gradually decreases with the increasing 

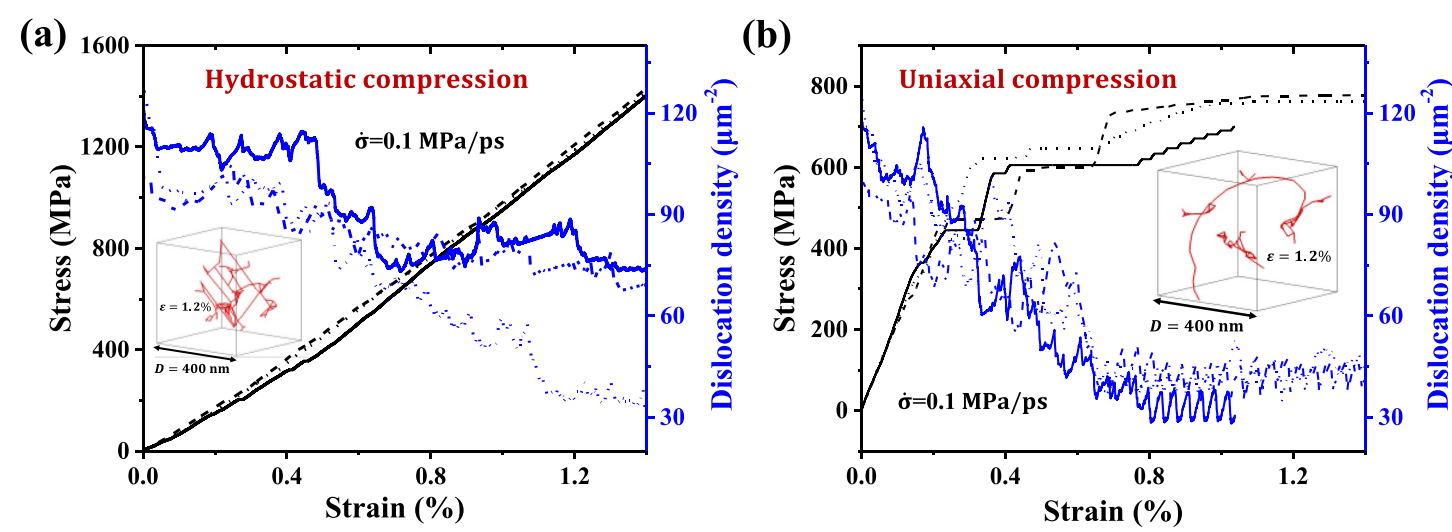

Figure 1. Stress (black line) and dislocation density (blue line) evolution for Cu Cubes with different initial structures under (a) hydrostatic compression test, and (b) uniaxial compression test.

hydrostatic pressure. In the case of uniaxial compressive loading, as shown in figure 1(b), however, the stressstrain curves exhibit the conventional pronounced step-like character [19], which suggests that the discrete strain burst events occur in a uncontrollable and stochastic way. After that, it can be observed that the stress reaches a critical stable value where the yield strength is obtained. The dislocation density is quite fluctuant, which is related to the operation and suppression of single-arm dislocation sources [3].

Another feature of the dislocation evolution under uniaxial compression is that the dislocation density decreases more rapidly to a stable state. It is because the dislocation located on the slip plane is activated via the effective resolved shear stress on the plane. For fcc single crystal with orientation $\langle 001\rangle$, the resolved shear stress is much larger in the case of uniaxial compression. By contrast, the dislocations can be hardly activated and tend to keep stable under the hydrostatic pressure, which leads to the insensitivity of the mechanical response to the confining pressure. The dislocation microstructures at the strain of $1.2 \%$ in the $\mathrm{Cu}$ sample with the same initial dislocation configuration are also illustrated in figure 1. It can be seen that all the dislocations are trapped on the slip plane in the hydrostatic compression test even though the stress is much higher ( 1200 MPa). While the operation of the single-arm dislocation source continues in the case of uniaxial loading, which leads to massive yielding. Next, the dislocation density and microstructure under hydrostatic pressure will be further analyzed.

\subsection{Size effect on the pressure sensitivity of dislocation density evolution}

From the above analysis, it can be seen that the mechanical responses appear to be insensitive to the confining pressure at small scales, which is further confirmed by analyzing the stress-strain curves for Cu samples of different size. However, the dislocation evolution shows some different characteristics. The further investigation is then focused on the evolution of dislocation density and microstructures in this section.

\subsubsection{Pressure sensitivity of dislocation density evolution}

The Cu samples with edge lengths ranging from 200 to $1200 \mathrm{~nm}$ are loaded under hydrostatic pressure with loading rate $0.5 \mathrm{MPa} \mathrm{ps}^{-1}$. The evolution of dislocation density with the increasing pressure is shown in figures 2(a)-(d), respectively. It can be seen that the evolution of dislocation density exhibits the size dependency under hydrostatic compression. For the $\mathrm{Cu}$ samples with the edge length 200 and $400 \mathrm{~nm}$, the dislocation density is relatively stable at the beginning and then decreases gradually with the increasing stress, exhibiting the nature of pressure sensitivity. It may be because the operation of dislocation sources requires larger critical shear stress in the sample of smaller size $[2,16]$ and dislocations tend to move out due to the image force by free surface when they are activated [3]. For larger samples $(D=800$ and $1200 \mathrm{~nm})$, after the initial decrease of dislocation density, which corresponds to the mechanical annealing process [4], the dislocation density can increase with the increasing hydrostatic pressure and achieve a relatively stable value finally. This process exhibits similar characteristic and insensitivity to the pressure, featuring a relatively stable dislocation density despite that the pressure keeps increasing.

As a reference, the dislocation evolution in Cu samples with the edge length 400 and $800 \mathrm{~nm}$ under uniaxial loading are also presented in figure 3. It can be seen that the evolution of dislocation density is quite different from that in hydrostatic pressure loading. In the early stage of plastic deformation, the stress when the massive dislocation multiplication occurs is relatively low, characterized by the appearance of stress steps in the stressstrain curves. The vertical lines in the curves of dislocation density evolution correspond to the dramatic dislocation activity. It indicates that dislocations are more easily activated and generate plenty of plasticity in this 
(a)

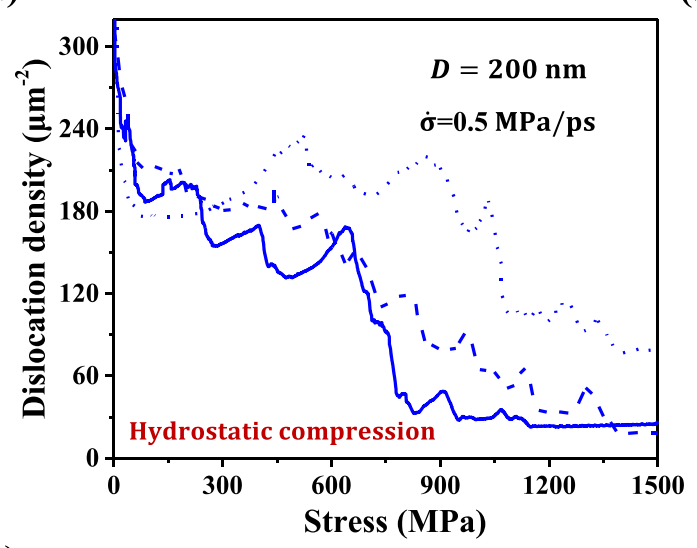

(c)

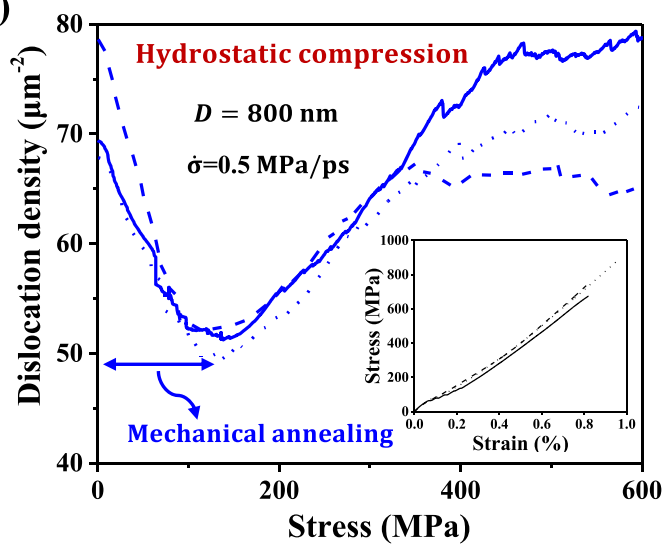

(b)

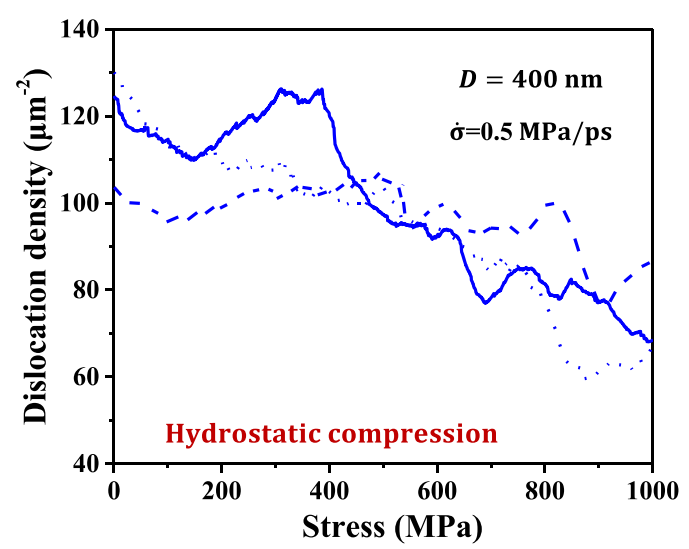

(d)

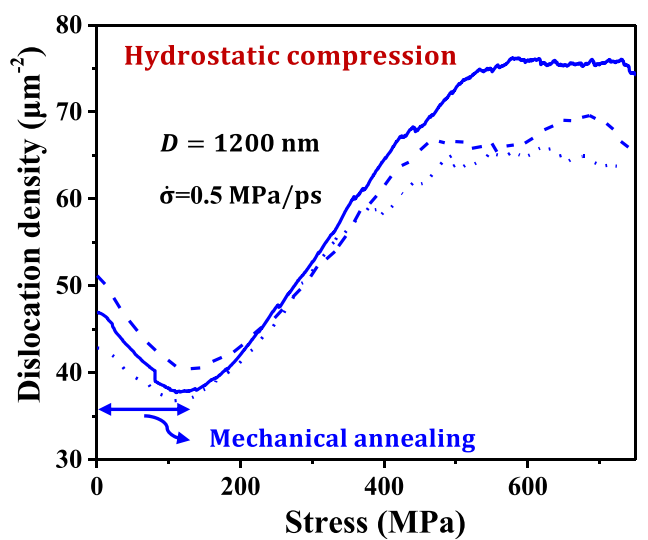

Figure 2. The evolution of dislocation density in Cu cubes with different edge lengths (a) $D=200 \mathrm{~nm}$, (b) $D=400 \mathrm{~nm}$, (c) $D=800 \mathrm{~nm}$ and (d) $D=1200 \mathrm{~nm}$ under hydrostatic loading.

(a)

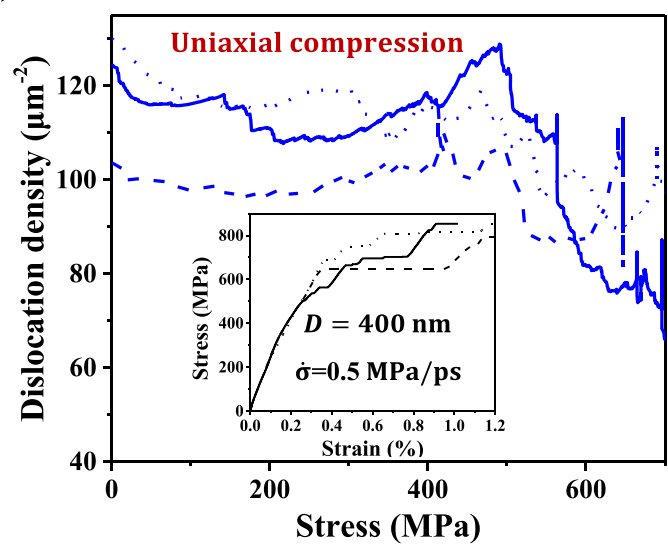

(b)

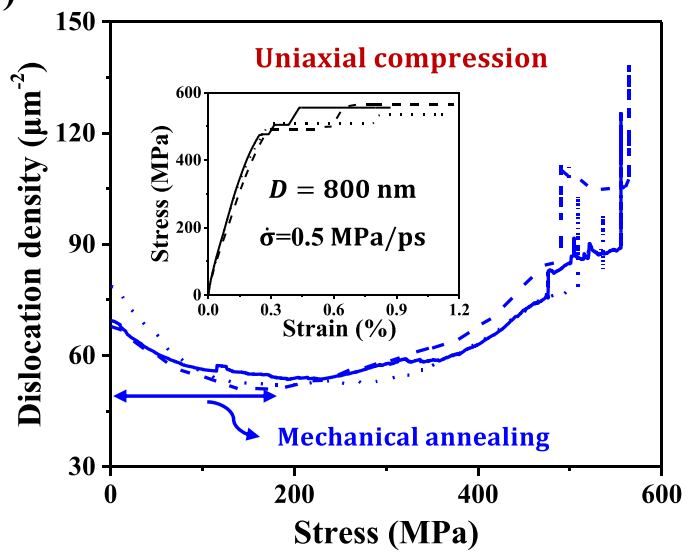

Figure 3. The evolution of dislocation density for $\mathrm{Cu}$ cubes with the edge lengths (a) $D=400 \mathrm{~nm}$ and (b) $D=800 \mathrm{~nm}$ under uniaxial compression.

case. The dislocation density continues to increase after the stress reaches the yield strength, resulting in a sample that is generally unable to withstand large loads under the uniaxial loading condition.

Two questions then arise, namely, why the sample size plays an important role on the evolution of dislocation density under hydrostatic compression especially when sample size becomes smaller $(D \leqslant 40 \mathrm{~nm})$, and why the evolution of dislocation density shows a significant difference between hydrostatic and uniaxial compression cases. 
(a)

$$
\sigma=200 \mathrm{MPa}
$$

$\sigma=400 \mathrm{MPa}$

$\sigma=800 \mathrm{MPa}$
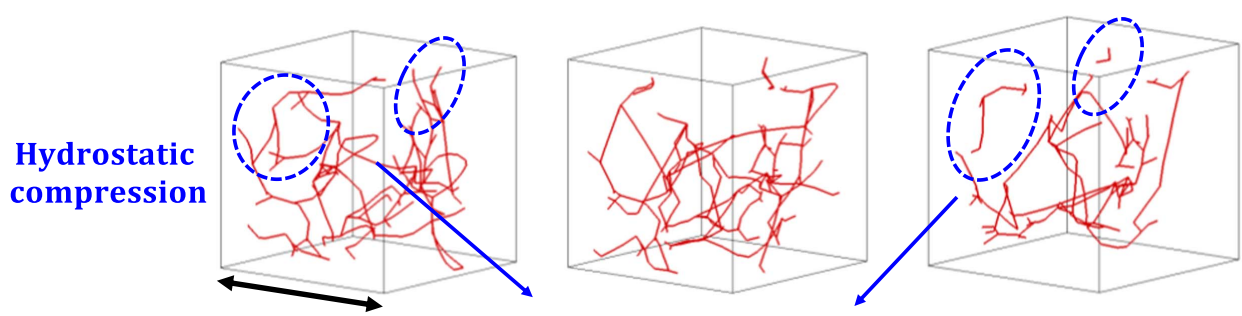

$D=400 \mathrm{~nm}$

Disruption of dislocation link

(b)
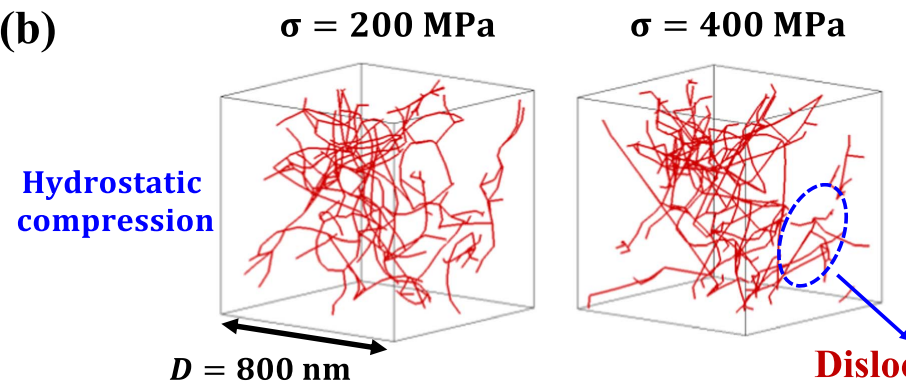

$\sigma=600 \mathrm{MPa}$

(c)

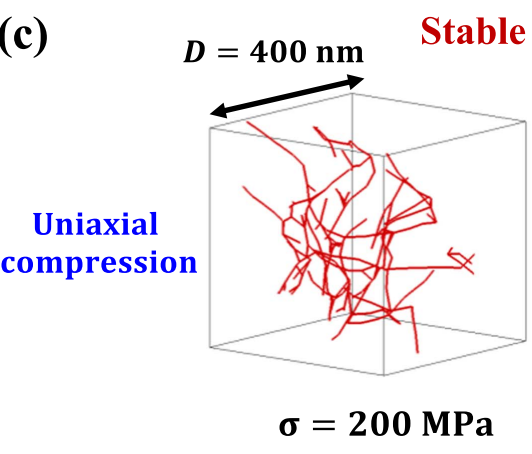

\section{Dislocation lock}

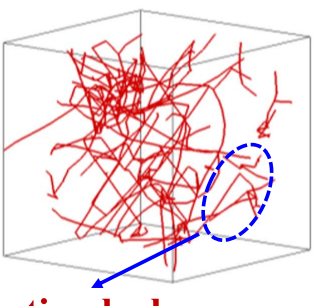

$\sigma=200 \mathrm{MPa}$
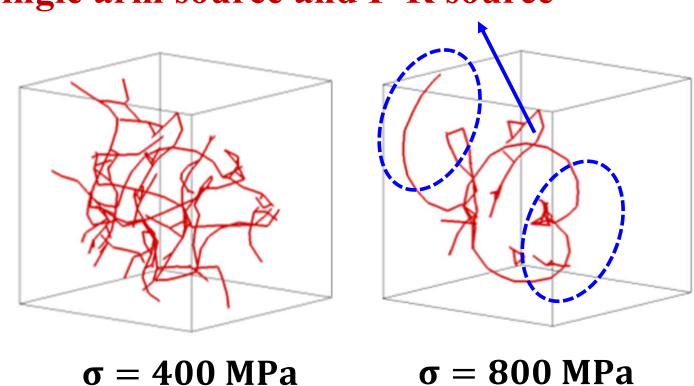

(d)

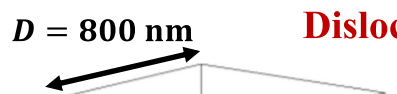

Uniaxial compression

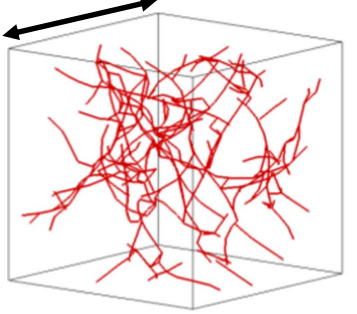

$\sigma=200 \mathrm{MPa}$

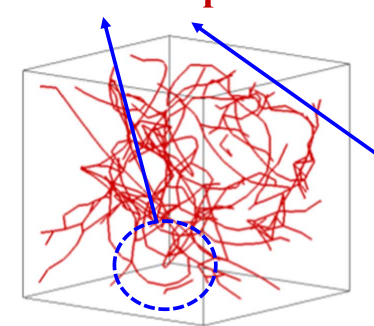

$\sigma=400 \mathrm{MPa}$

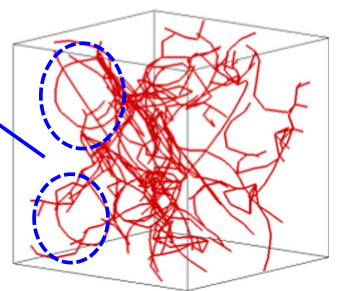

$\sigma=535 \mathrm{MPa}$

Figure 4. The evolution of dislocation microstructures for Cu cubes with different edge lengths (a) $D=400 \mathrm{~nm}$ and (b) $D=800 \mathrm{~nm}$ under hydrostatic compression, (c) $D=400 \mathrm{~nm}$ and (d) $D=800 \mathrm{~nm}$ under uniaxial compression.

\subsubsection{Dislocation microstructure analysis}

The dislocation microstructures are further analyzed to answer the above questions. As shown in figures 4(a) and (b), in the case of hydrostatic compression, for $\mathrm{Cu}$ samples with the edge length $400 \mathrm{~nm}$, the dislocations are intermittently activated and the links between dislocations are destroyed as the pressure increases. At the same time, new stable dislocation links are difficult to form before these dislocations are driven out of the samples. The remaining dislocations are more stable and require a higher activation stress. As a result, the dislocation density gradually decreases with the increasing stress, leading to the pressure sensitivity in the evolution of dislocation density. By contrast, for Cu sample as large as $800 \mathrm{~nm}$ as shown in figure 4(b), many of the generated dislocations can form new junctions and tangles as new sources rather than slip out immediately. Therefore, the dislocation density can increase again after the initial mechanical annealing process occurs. The reconstruction of jammed dislocation network is quite stable, which is the key to be responsible for the pressure insensitivity.

Moreover, the dislocation microstructures for $\mathrm{Cu}$ samples with the edge length 400 and $800 \mathrm{~nm}$ under uniaxial compression are also presented in figures 4(c) and (d), respectively. For Cu sample with the edge length 
(a)

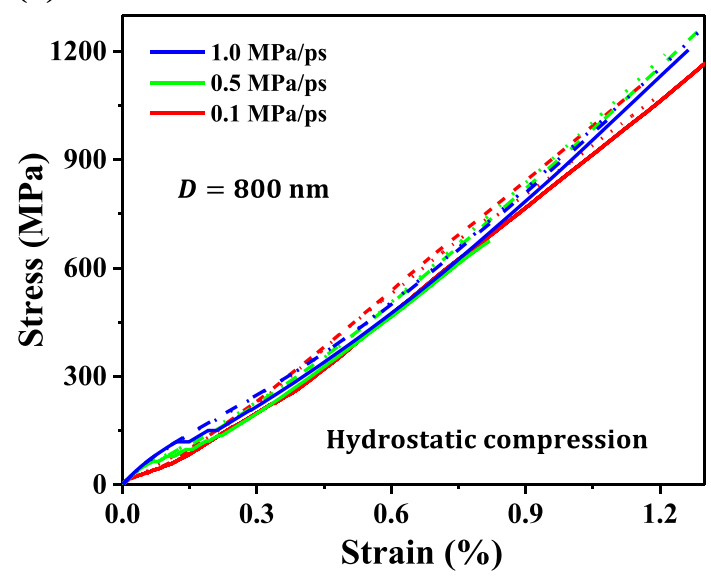

(b)

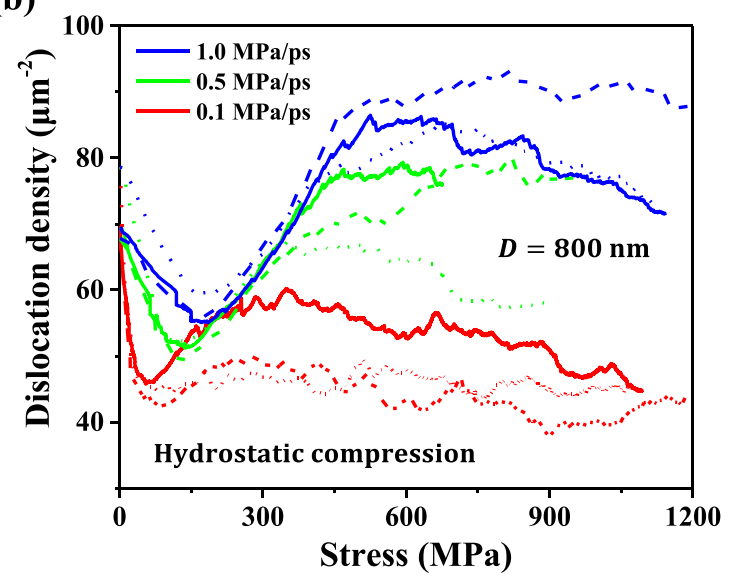

Figure 5. (a) Stress-strain curves for Cu samples under different pressure loading rates, and (b) the evolution of dislocation density.

$400 \mathrm{~nm}$, see figure 4(c), some stable single-arm dislocation sources and Frank-Read (F-R) sources can form eventually. These stable dislocation sources continuously sweep the slip plane and generate a large amount of plastic deformation to maintain a steady plastic flow. Similarly, in Cu sample with the edge length $800 \mathrm{~nm}$, it can be seen that with the increase of external load, more dislocation sources are activated, which leads to the dislocation multiplication. When the stress reaches the yield strength of $535 \mathrm{MPa}$, the dislocation multiplication continues. For the hydrostatic loading considered here, the internal dislocations are hardly activated under the confining pressure. Thus, a number of dislocation locks are formed and quite stable, which can serve as the obstacle for dislocation motion. This may be one possible reason why materials can always reach much higher pressure level under shock loading, in which the region of the material that has been deformed will normally enter into a hydrostatic state [9], so that the movement of internal dislocation is suppressed and the stress relaxation can be hardly achieved.

\subsection{Effect of hydrodynamic loading rate}

In order to study the rate effect, the mechanical responses for the $\mathrm{Cu}$ samples with the edge length $800 \mathrm{~nm}$ as well as the evolution of dislocation density under different pressure loading rates are presented in figure 5 . The results in figure 5(a) further confirm that the mechanical responses are insensitive to the confining pressure, even under different pressure loading rates, which is quite different from the rate-dependent mechanical behavior under uniaxial compression [20]. However, the evolution of dislocation density shows a significant rate effect. After the initial mechanical annealing process, the dislocation density starts to increase due to the activation of dislocation sources and becomes approximately stable finally. The stable dislocation density is increasing with the increase of pressure loading rates.

Correspondingly, the dislocation microstructures in the $\mathrm{Cu}$ sample with the same initial dislocation configuration, which is at the hydrostatic pressure of $1000 \mathrm{MPa}$, are shown in figure 6. Firstly, the typical structural characteristics of dislocations are quite different from those of uniaxial compression, in which most of the dislocation lines are curved when the operation of single-arm or F-R dislocation sources occurs. Conversely, the dislocation structures under hydrostatic pressure are always straight with sharp edges and corners, which is consistent with the dislocation morphology observed in the silicon sample under the pressure of $1500 \mathrm{MPa}$ [21]. Furthermore, by analyzing the evolution of dislocation structures under different loading rates, the rate effect on the dislocation density can be attributed to the transform of dislocation structures from curved lines to straight ones that are the final stable morphological structures of dislocation under hydrostatic pressure. A faster increasing rate in the pressure makes it more probable to form the straight dislocation lines before the activated curved dislocations slip out from the samples. As a result, the stable dislocation structures tend to concentrate in the central region. A high hydrostatic pressure can effectively eliminate the effect of image force and keep the dislocations trapped inside the $\mathrm{Cu}$ samples. With the increase of hydrostatic pressure, however, the forces acting on the dislocations are still not sufficient to break the strong links between the internal dislocations so that the dislocations cannot generate enough plasticity under hydrostatic loading. As a result, the mechanical response does not exhibit an obvious rate effect. 
(a)

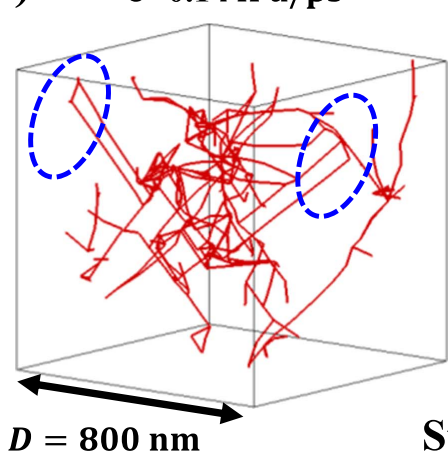

(b)

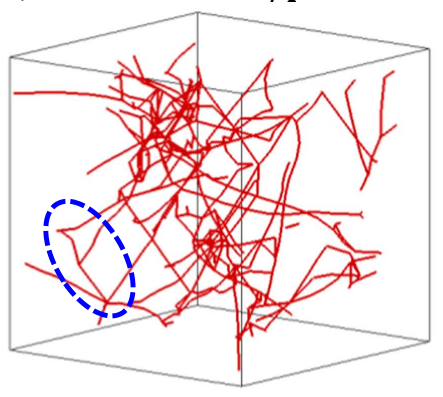

(c)

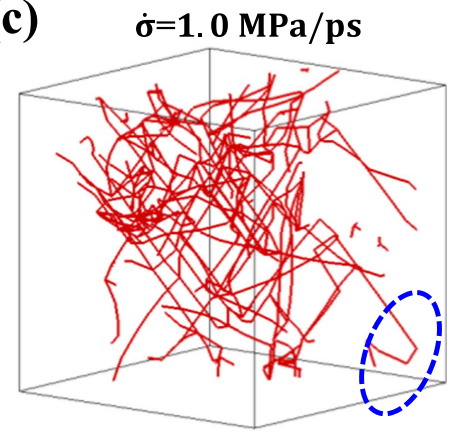

Straight dislocations with sharp edges and corners

Figure 6. Dislocation structures in Cu sample with the edge length $800 \mathrm{~nm}$ at the hydrostatic pressure of $1000 \mathrm{MPa}$ and the loading rates of (a) $0.1 \mathrm{MPa} \mathrm{ps}^{-1}$, (b) $0.5 \mathrm{MPa} \mathrm{ps}^{-1}$, and (c) $1.0 \mathrm{MPa} \mathrm{ps}^{-1}$.

\section{Concluding remarks}

In summary, the dislocation density of $\mathrm{Cu}$ samples as well as the corresponding microstructures have been examined under hydrostatic compression, and compared with the results under uniaxial compression. The effects of loading mode, sample size and loading rate have been explored on the mechanical responses. It is found that the dislocation structures are less activated under the hydrostatic compression as compared with the uniaxial compression. There exists a size-dependent transition in the evolution of dislocation density, which exhibits the pressure sensitivity as the sample size becomes smaller than a specific value $(D \leqslant 40 \mathrm{~nm})$. Although the rate-dependent stress-strain relationship is not observed under the hydrostatic compression, the evolution of dislocation density exhibits a significant rate dependence. This is because a faster increase in stress makes it more rapid to suppress the effect of image forces, and easier to keep the dislocations trapped inside the samples. Furthermore, the typical characteristics of dislocation structures under hydrostatic compression are analyzed. It is found that the straight dislocations with sharp edges and corners are quite stable under the confining pressure. The findings reported here could provide a better understanding on the elastoplastic responses of metallic samples at microscale.

\section{Acknowledgments}

This work is supported in part by the National Natural Science Foundation of China through Grant No. 11132006,11302115 and 11232003 .

\section{ORCID iDs}

Jianqiao Hu (10) https://orcid.org/0000-0003-2849-4252

\section{References}

[1] Weinberger C R, Aubry S, Lee S-W and Cai W 2009 IOP Conf. Ser.: Mater. Sci. Eng. 3012007

[2] Zhou C, Beyerlein I J and LeSar R 2011 Acta Mater. 59 7673-82

[3] Cui Y, Lin P, Liu Z and Zhuang Z 2014 Int. J. Plast. 55 279-92

[4] Shan Z W, Mishra R K, Syed Asif S A, Warren O L and Minor A M 2008 Nat. Mater. 7 115-9

[5] Liu Z L, Liu X M, Zhuang Z and You X C 2009 Scr. Mater. 60 594-7

[6] Ryu I, Cai W, Nix W D and Gao H 2015 Acta Mater. 95 176-83

[7] Shehadeh M A, Bringa E M, Zbib H M, McNaney J M and Remington B A 2006 Appl. Phys. Lett. 89171918

[8] Kattoura M and Shehadeh M A 2014 Phil. Mag. Lett. 94 415-23

[9] Hu J, Liu Z, Chen K and Zhuang Z 2017 Comput. Mater. Sci. 131 78-85

[10] Yuan F and Wu X 2014 Comput. Mater. Sci. 85 8-15

[11] Chen Z, Jiang S, Sewell T D, Gan Y, Oloriegbe S Y and Thompson D L 2014 J. Appl. Phys. 116233506

[12] Cui Y, Liu Z and Zhuang Z 2015 J. Mech. Phys. Solids 76 127-43

[13] Gao Y, Liu Z L, You X C and Zhuang Z 2010 Comput. Mater. Sci. 49 672-81

[14] Wang Z Q, Beyerlein I J and Lesar R 2007 Phil. Mag. 87 2263-79

[15] Wang Z Q, Beyerlein I J and LeSar R 2009 Int. J. Plast. 25 26-48

[16] Zhou C, Biner S B and LeSar R 2010 Acta Mater. 58 1565-77

[17] Lee S-W, Han S M and Nix W D 2009 Acta Mater. 57 4404-15

[18] Jennings A T, Burek M J and Greer J R 2010 Phys. Rev. Lett. 104135503 
[19] Kiener D and Minor A M 2011 Acta Mater. 59 1328-37

[20] Jennings A T, Li J and Greer J R 2011 Acta Mater. 59 5627-37

[21] Wzorek M, Czerwinski A, Ratajczak J, Misiuk A and Kątcki J 2007 Vacuum 81 1229-32 